\title{
Criminologie
}

\section{La sécurité privée : le phénomène, la contreverse, l’avenir}

\section{Maurice Cusson}

Volume 31, numéro 2, automne 1998

La sécurité privée

URI : https://id.erudit.org/iderudit/017417ar

DOI : https://doi.org/10.7202/017417ar

Aller au sommaire du numéro

Éditeur(s)

Les Presses de l'Université de Montréal

ISSN

0316-0041 (imprimé)

1492-1367 (numérique)

Découvrir la revue

Citer cet article

Cusson, M. (1998). La sécurité privée : le phénomène, la contreverse, l'avenir. Criminologie, 31(2), 31-46. https://doi.org/10.7202/017417ar

\section{Résumé de l'article}

This article deals with four themes on private security. First, private security is defined as a protection supplied to organizations targeting the specific needs of any given organization. Second, the expansion of private security is seen as resulting from the convergence between a real demand for security and a superior supply than the one offered by the police. Third, the article deals with questions regarding private security involving private live-Finally, it is argued that the future of private security lies in two trends : (1) technologicial integration and (2) sophisticated planning and problem solving. 
This article deals with four themes on private security. First, private security is defined as a protection supplied to organizations targeting the specific needs of any given organization. Second, the expansion of private security is seen as resulting from the convergence between a real demand for security and a superior supply than the one offered by the police. Third, the article deals with questions regarding private security involving private live. Finally, it is argued that the future of private security lies in two trends: (1) technologicial integration and (2) sophisticated planning and problem solving.

\section{INTRODUCTION}

Les criminologues ne se rendent pas tous compte jusqu'à quel point les effectifs de la sécurité privée sont présents dans la prévention de la délinquance et des incivilités. En effet, ils font face aux vols, fraudes, actes de vandalisme et désordres publics, dans les supermarchés, les grands magasins, les centres commerciaux, les hôpitaux. Dans ces espaces où se pressent des milliers de gens, il leur incombe de gérer les problèmes posés par les personnes ivres, les vandales et les malades mentaux en crise. Quand les commerçants et les administrateurs ont pris conscience de la menace que représentait l'augmentation des larcins, des fraudes et des incivilités, il leur a paru évident que la police publique se consacrait à des problèmes plus graves et ne pouvait leur offrir les services sur mesure qu'ils en attendaient. La sécurité privée apparut alors comme un recours. Ainsi s'imposa, au cours du $\mathrm{XX}^{\mathrm{e}}$ siècle, un mode de contrôle social inédit qui, parce qu'il était soumis aux règles de l'économie de marché, devait pratiquer des prix compétitifs et répondre aux attentes du client sans interférer dans ses opérations. Motivés par la demande et talonnés par la concurrence, les experts en sécurité ont alors inventé une version empirique de prévention situationnelle.

La sécurité privée reste une nébuleuse mal comprise et mal jugée. D'abord, il importe d'en appréhender la nature. Ensuite, on montrera que c'est le marché qui lui fournit sa dynamique propre. Puis, on fera le point

1. Professeur titulaire à l'École de criminologie de l'Université de Montréal, C.P. 6128, Succursale centre-ville, Montréal (Québec), Canada, H3C 3J7. 
sur les inquiétudes qu'elle suscite. Enfin, seront évoquées deux tendances porteuses d'avenir dans le secteur.

\section{LE PHÉNOMÈNE}

Dans cette première partie, la nature de la sécurité privée sera approchée : 1) par un essai de définition ; 2) par l'examen de ses fonctions.

\section{Définition}

L'expression sécurité privée ne soulève pas de difficulté quand il est question d'une agence de gardiennage chargée de la surveillance d'un centre commercial ou du service de sécurité de la compagnie IBM, par exemple. Mais l'usage s'est imposé d'inclure dans la sécurité privée les services affectés à la protection de sociétés d'État, comme Hydro-Québec, et de services publics, comme les hôpitaux ou les aéroports. Avec Brodeur (1995), nous pourrions toujours qualifier d'hybrides ces appareils mi-privés mi-publics ; mais que dire des fonctionnaires chargés de la sécurité dans les édifices gouvernementaux ? Ils relèvent carrément de l'État et pourtant nous sommes encore portés à les assimiler à la sécurité privée. Les intéressés eux-mêmes le pensent aussi. La preuve : ils sont souvent membres d'associations de sécurité privée, comme la Société canadienne de sûreté industrielle.

Qu'ont en commun tous les organismes que l'usage courant place sous le chapeau de la sécurité privée ? La réponse pourrait être qu'ils offrent une sécurité ciblée, une protection qui profite en propre à un client particulier ou à un site déterminé. Alors que la police publique a pour mission de faire régner la sécurité partout, l'agence ou le service de sécurité ne protège que les intérêts de son client et ne rend compte qu'à lui. La sécurité privée est donc une sécurité particulière : elle ne s'intéresse qu'aux besoins du client tels qu'il les définit lui-même. Elle se caractérise par un désir, motivé par le profit, de répondre à ses souhaits et par une mission circonscrite : assurer la sécurité de tel site, de telles personnes ou de tel réseau, à l'exclusion de tout autre site, personne ou réseau. De son côté, la sécurité publique assume des responsabilités plus étendues et plus diffuses; elle étend son parapluie protecteur à toute la collectivité et fait respecter partout les lois en appréhendant les délinquants et en les traduisant en justice.

Une autre manière de saisir la spécificité de la sécurité privée est de la comparer à la police. Loubet del Bayle (1992 : 20) définit cette dernière en ces termes : il y a fonction policière lorsque des aspects majeurs de la régulation sociale sont assurés par une institution agissant au nom du groupe et ayant la possibilité « d'user en ultime recours de la force physique ». Il ajoute (p. 23), que cette fonction est consubstantielle à l'organisation politique. Par 
opposition, trois caractéristiques de la sécurité privée sautent aux yeux : 1) elle n'agit pas au nom du groupe, mais de son client ; 2) elle ne dispose qu'exceptionnellement du pouvoir d'user de la force, avant tout elle surveille et prévient ; 3) elle ne relève pas du politique, étant régie principalement par les lois du marché. Ces considérations débouchent sur une définition :

Par sécurité privée ou particulière, nous entendons l'ensemble des biens et services servant à la protection des personnes, des biens et de l'information que des spécialistes motivés par le profit offrent à des organisations en vue de répondre à leurs besoins particuliers ${ }^{2}$.

Cette définition souligne d'abord la finalité première de la sécurité privée : la protection des personnes, des biens et de l'information contre tout danger. Les plus gros demandeurs de sécurité privée sont moins des individus que des organisations : commerces, manufactures, établissements d'enseignement, ministères et entreprises parapubliques. Le mot spécialiste sert à exclure de notre propos l'autoprotection et la sécurité diffuse intégrées au fonctionnement quotidien des établissements : le petit commerçant observant du coin de l'œil un client, l'enseignant surveillant la salle de récréation et la secrétaire qui, par sa simple présence, empêche le vol de matériel de bureau.

\section{Les fonctions}

L'observateur reste dérouté devant l'extraordinaire diversité des biens et services offerts sur ce marché. Une manière de mettre un peu d'ordre dans ce fatras serait de classer chaque élément selon sa fonction, c'est-à-dire selon sa contribution à la sécurité. Six fonctions seront distinguées.

La surveillance. Il s'agit de garder un site sous observation de manière à détecter les signes de danger ou de malveillance. L'importance de cette fonction est mise en évidence par le nombre de termes utilisés pour désigner les hommes qui l'exercent: garde de sécurité, gardien, vigile, veilleur de nuit... De plus en plus, ces surveillants s'appuient sur des moyens techniques pour prolonger leur vision : alarmes, détecteurs, caméras, éclairage, ceil magique, étiquette électronique, miroir, lecteur laser de code...

Le contrôle des accès et l'obstacle à l'intrusion. Il s'agit de filtrer les entrées sur un site, d'empêcher que des intrus ou des indésirables ne s'y trouvent en position de poser un acte malveillant, et de protéger physiquement les sites et les cibles. Contrôle d'accès et surveillance sont souvent associés.

2. La définition proposée par Fourcaudot $(1988: 16)$, qui sert de référence au Québec, précise, elle, que la sécurité privée est «l'ensemble des activités et des mesures visant la protection des personnes, des biens et de l'information fournie dans le cadre d'un marché compétitif orienté vers le profit et où les pourvoyeurs n'assurent pas, au regard de la loi, des responsabilités de fonctionnaire au service du gouvernement ». 
Les moyens matériels et techniques d'empêcher les intrusions ne manquent pas : portes, serrures, clôtures, murs, barrières, grillages, vitrages, cartes d'accès, badges, systèmes d'ouverture électronique...

L'investigation. Les détectives privés et les investigateurs des agences de sécurité recherchent les auteurs de délits. Cette fonction est réalisée par l'interrogatoire, la filature, l'installation de caméras cachées, l'infiltration...

Le transport de fonds. Rare secteur où les gardes sont armés.

L'intervention. C'est l'action menée à la suite de la détection d'un incident, d'un danger, d'un intrus ou d'un délinquant. L'intervention est différente selon que le contrevenant est un employé de l'entreprise visée, un client, un intrus occasionnel ou un récidiviste. Les employés pris la main dans le sac s'exposent à des sanctions disciplinaires, au congédiement, aux poursuites civiles et aux poursuites pénales. Quand un voleur à l'étalage occasionnel est attrapé, on se contente le plus souvent de récupérer l'objet dérobé et de lui donner un avertissement.

Le renseignement. C'est la collecte et l'analyse d'informations utiles pour la planification et l'adaptation des mesures de sécurité.

\section{LE MARCHÉ ET LA DYNAMIQUE DE LA SÉCURITÉ PRIVÉE}

La sécurité privée est soumise à la logique du marché, entendue comme un espace symbolique ou sont confrontées toutes les offres et demandes d'un bien ou service. Les services de sécurité interne des sociétés d'État échappent en partie aux lois du marché, mais en partie seulement, car s'y fait sentir la concurrence des agences externes. Ce marché est dominé par une demande de prévention : la répression n'y occupe qu'une place effacée. Ce qui s'y transige, c'est d'abord et avant tout des moyens de surveillance, de contrôle d'accès et de renforcement des cibles.

Pour rester en affaire, les acteurs de la sécurité privée doivent trouver le moyen d'assurer la protection des personnes, des biens et de l'information de leurs commettants, au moindre coût, sans entraver le déroulement des opérations normales et sans empiéter dans l'intimité des gens. Leur mission ne se réduit pas à préserver la rentabilité de l'entreprise en limitant ses pertes, ils ont aussi pour mandat de mettre à l'abri du danger les personnes et les biens de tous ceux qui se trouvent sur les sites à protéger : employés, acheteurs (dans un magasin) et visiteurs. Tous doivent pouvoir vaquer à leurs occupations en toute quiétude.

Pour expliquer pourquoi la sécurité privée est devenue, au cours du $\mathrm{XX}^{\mathrm{e}}$ siècle, un joueur majeur dans la prévention du crime, le raisonnement doit s'appuyer autant sur la science économique que sur la criminologie. 
En stricte logique économique, l'existence même d'un marché de la sécurité d'une certaine ampleur présuppose une demande elle-même tributaire d'une insécurité bien réelle. Et si les acteurs économiques ne paient pas volontiers de leurs deniers ce qu'ils peuvent obtenir gratuitement, pourquoi ont-ils déboursé pour une sécurité que l'État fournit en principe sans frais ? C'est que l'expansion du marché de la sécurité résulte de la rencontre d'un besoin réel de sécurité ressenti dans le monde du commerce et d'une offre privée plus intéressante qu'ailleurs.

La réalité des menaces qui pèsent sur les demandeurs de sécurité apparaît clairement à l'examen, même sommaire, de la victimisation des entreprises. Il est connu que la criminalité a fortement augmenté à partir des années 1960. Ce qui l'est moins, c'est que le secteur commercial subit plus que sa part de victimisation, étant frappé de plein fouet par la croissance de la criminalité des 35 dernières années. Le sondage international de victimisation des entreprises réalisé en 1993-94 dans huit pays européens montre que les taux de cambriolage dans le commerce de détail sont partout 10 fois plus élevés que dans les résidences (van Dijk et Terlouw, 1996).

Quelle est la nature des délinquances et malveillances qui s'attaquent aux commerces ? Les résultats obtenus par Ocqueteau et Pottier (1995a) dans les hypermarchés peuvent être ajoutés à ceux de Phillips et Cochrane (1988) dans des centres commerciaux, de Poyner et Webb (1992) dans le marché central de Birmingham, et de Bellot et Cousineau (1996) dans le métro de Montréal, pour illustrer l'étonnante variété de délinquances et d'incivilités auxquelles font face les agents de sécurité. Six types seront distingués :

1. les vols à l'étalage et les fraudes dont des clients ou des visiteurs se rendent coupables;

2. les incivilités et les nuisances : mendicité, ivresse, consommation de drogues, vandalisme, attroupements, tapage et bousculades ;

3. les vols commis par les employés ;

4. les vols commis à l'encontre de la clientèle : vols de sac à main, vols à la tire ;

5. les vols par effraction ;

6. les violences : attaque à main armée, agression contre client, incendie volontaire, bombe.

Après avoir épluché presque mille dossiers d'intervention d'une agence de Montréal qui assure la protection de pharmacies de grandes surfaces, Gagnon (1995) aboutit aux chiffres suivants : $91 \%$ des interventions colligées portent sur des vols à l'étalage, $85 \%$ des pertes sont de moins de $25 \$, 90 \%$ 
des suspects sont non violents et $76 \%$ collaborent avec l'agent qui les interpelle. C'est dire que dominent massivement dans ce contentieux les petites affaires qui se règlent en douce parce que le délinquant s'incline et ne fait pas d'esclandre. Dès que la gravité se hausse d'un cran, l'agent fait appel à la police.

Ces faits nous révèlent que le principal problème qui a stimulé la demande de sécurité est le volume important de petits délits et d'incivilités s'abattant sur les commerces et sur les espaces semi-publics. Pour préserver la qualité de vie de ces milieux, il fallait soit prévenir ces agissements, soit les gérer en douceur. Mais pourquoi les commerçants et directeurs d'établissements ont-ils préféré faire appel à la sécurité privée plutôt qu'à la police et à la justice ? Et encore, pourquoi n'ont-ils pas voulu s'en remettre à des contrôles sociaux plus informels? Tout simplement parce que ces recours étaient trop peu disponibles. En effet, au fil des ans, les forces de l'ordre et les tribunaux sont devenus de plus en plus sourds à leurs appels et se sont progressivement désengagés du champ de la petite et moyenne délinquance. Au Québec, Gagnon (1995), de même que Tremblay et Cousineau (1996), ont analysé plusieurs centaines de dossiers dans une agence contractuelle et un service interne de sécurité pour distinguer les cas qui font l'objet d'une plainte formelle à la police de ceux qui ne le font pas. Ne sont signalés à la police que les suspects qui ont fait subir à l'entreprise une perte relativement importante, qui refusent de coopérer, qui sont des récidivistes et qui ne sont pas des employés de l'entreprise. En Île-de-France, Ocqueteau et Pottier (1995b : 72) constatent que $95 \%$ des interpellations par les vigiles pour vols commis dans un hypermarché échappent totalement à la police. Les rares vols à l'étalage que l'on finit par signaler à l'attention policière présentent une ou plusieurs des caractéristiques suivantes : 1) la valeur du bien volé est relativement élevée ; 2) le voleur opère avec un ou des complices ; 3) il s'exécute avec une habileté qui donne à penser qu'il est un récidiviste ; 4) il est un récidiviste ; 5) il se rebelle et menace les agents ; 6) il nie les faits et refuse de décliner son identité.

Par la force des choses, il s'est développé une division du travail de facto entre la répression publique et la sécurité privée. La première se réserve les délits commis sur la voie publique, la criminalité de violence, les vols graves, le crime organisé et les affaires de drogue ; la seconde prend en charge les incivilités et la délinquance mineure ou modérément grave commise sur les sites ou à l'encontre des entreprises et autres organisations. Comme la sécurité particulière coûte beaucoup moins cher que la police, cette division du travail s'impose même aux administrations publiques. Les ministères et les régies d'État font volontiers appel à des agences de gardiennage pour surveiller leurs sites. La raison? Selon la revue Canadian Security d'octobre 
1995, le salaire annuel moyen d'un policier est deux fois plus élevé que celui d'un garde de sécurité.

Même quand elle est accessible, la solution pénale publique ne va pas sans inconvénients. Elle est peu adaptée à la spécificité de chaque site, car les policiers, les procureurs et les juges ont tendance à réagir aux problèmes de manière légaliste et stéréotypée. Autre désavantage, l'action pénale risque d'être coercitive et stigmatisante. En effet, quand l'appareil répressif est mis en branle, la main de la justice s'abat brutalement sur le suspect ; la victime perd le contrôle du processus et elle risque d'être éclaboussée par l'opprobre qui frappe le coupable. Rares sont les dirigeants d'entreprises qui apprécient que les turpitudes de leurs employés soient étalées sur la place publique. Ils préfèrent, autant que possible, gérer le problème discrètement par la réprimande, la suspension, la demande de remboursement ou le congédiement.

On comprend alors pourquoi les organisations payent pour obtenir, sur une base privée, la protection souple, discrète et adaptée que la justice publique ne peut leur offrir.

\section{INQUIÉTUDES ET CONTROVERSES}

La légitimité de la sécurité privée reste sujette à controverse. L'idée que le marché puisse s'immiscer dans ce qui est considéré comme une juridiction exclusive de l'État paraît détestable à plusieurs. Et l'irruption de la logique du profit dans une activité traditionnellement justifiée par le bien commun est mal vue. Les griefs visant ceux que d'aucuns appellent vigiles ne sont pas mineurs : menaces aux droits et libertés, service d'intérêts particuliers au détriment des intérêts collectifs, pratiques d'une légalité douteuse.

\section{Big brother ?}

Le sujet d'inquiétude le plus grave tient aux redoutables moyens que fournit la technologie moderne à la sécurité privée. Ne pourrait-elle pas nous entraîner vers une société panoptique de surveillance omniprésente et vers un univers totalitaire dans lequel l'intimité des gens sera constamment menacée d'intrusion? Ne risque-t-elle pas de devenir une puissance qui sera encore plus difficile à contrôler que ne l'est la police (Christie, 1993) ?

Il est vrai que la surveillance est une activité centrale en sûreté industrielle. Et comme le soulignait Reiss (1987 : 20), le paradoxe, dans cette situation, est que, pour protéger notre espace privé, nous donnons à des spécialistes le pouvoir d'empiéter dans cette même vie privée. Cela dit, dans leurs utilisations les plus courantes, les systèmes de surveillance sont installés ostensiblement dans des endroits publics, comme les commerces. On ne voit pas en quoi des caméras qui balayent l'intérieur d'un magasin violent les droits et libertés individuelles. 
Dans nos États, les services de sécurité jouissent-ils de pouvoirs exorbitants ? Il faut d'abord savoir qu'ils sont limités par les lois et les chartes. Au Québec, la grande majorité des agents de sécurité n'ont pas plus de pouvoirs que ceux reconnus par la loi à un simple citoyen qui prend un voleur sur le fait. Ils n'ont ni le droit de fouiller ni celui d'user de la force (Gagnon, 1995). Le Code civil protège le citoyen contre les atteintes à sa vie privée et il balise très strictement le pouvoir des agences de sécurité. Ces dernières s'exposent à des poursuites judiciaires si elles font intrusion dans la vie privée.

Les agences et services de sécurité doivent aussi compter avec d'autres pouvoirs qui leur font contrepoids : les polices publiques, les gouvernements, le pouvoir judiciaire, les mass-media et même les autres agences. En effet, les entreprises de sécurité se font concurrence. Si l'une d'elles se discrédite en se livrant à des abus, d'autres ne seront que trop contentes d'en profiter pour s'accaparer sa part de marché. Le pouvoir politique craint beaucoup plus la police que la sécurité privée, comme le montrent les concessions que les syndicats policiers arrachent aux politiciens. Il est facile de faire jouer la police contre la sécurité privée. L'inverse est beaucoup plus difficile. Il se pourrait même que le marché de la sécurité nous ait épargné un État policier. En effet, sans son apport, il est vraisemblable que l'État aurait consacré des ressources énormes pour lutter contre le vol à l'étalage, les fraudes et toutes les menaces qui pèsent sur les corporations. Cela aurait signifié une extension des effectifs policiers et l'octroi de pouvoirs encore plus étendus à l'État. La démocratie et les libertés sont mieux protégées par un grand nombre d'entreprises de sécurité, obéissant aux dures lois du marché et tenues en laisse par les pouvoirs publics, que par des forces de police géantes qui se seraient réservé la totalité des actions contre le crime.

Cela dit, des entreprises spécialisées dans la surveillance, le renseignement et la filature seront inévitablement tentées d'en abuser ; c'est pourquoi la vigilance s'impose.

\section{Intérêt particulier, déplacement et bien commun}

Selon South (1988) les gens de la sécurité privée ne se lancent pas en affaires pour servir le bien commun, mais pour promouvoir les intérêts de leurs clients. Même s'ils réussissent à prévenir le crime sur un site, ils contribuent assez peu à la sécurité collective car une part importante des délits prévenus ne sont que déplacés (voir aussi Shearing et Stenning, 1981).

Il est vrai que l'intérêt bien compris des intervenants en sécurité industrielle ne dépasse guère celui de leur client ou employeur et que les intérêts du client peuvent entrer en conflit avec ceux du passant. Mais se pourrait-il qu'ils servent, sans le vouloir, le bien commun en servant des intérêts particuliers ? Toute initiative privée de sécurité qui réussit à prévenir des 
crimes sans les déplacer tous contribue, même de manière minime, à limiter le nombre des crimes à l'échelle de la société globale. Si le déplacement est toujours possible, il n'est jamais total. Qui plus est, il arrive que des mesures de prévention situationnelle utilisées en sécurité privée fassent baisser les vols non seulement dans les sites protégés, mais aussi dans leurs environs immédiats qui, eux, ne jouissent pas d'une protection particulière. C'est donc dire que les bénéfices de la sécurité privée se diffusent quelquefois de ceux qui payent à ceux qui ne payent pas ${ }^{3}$. Si on convient que la sécurité collective n'est rien d'autre que la somme des sécurités individuelles, alors un système de protection privé aménagé sur un site contribuera à la sécurité collective s'il prévient plus de délits qu'il n'en déplace. $\mathrm{Si}$, en outre, les bénéfices de mesures préventives privées se diffusent, la contribution sera encore plus évidente. L'apport principal de la sécurité privée à la sécurité collective se réalise par la réduction du nombre des cibles intéressantes et vulnérables offertes aux délinquants potentiels : ces derniers commettent moins de délits car un moins grand nombre d'opportunités criminelles s'offrent à eux.

\section{Une protection réservée aux riches ?}

L'argument sejon lequel la sécurité privée est un luxe auquel les pauvres n'ont pas accès contient certainement une part de véritét ${ }^{4}$. D'autant plus que les pauvres sont victimisés plus souvent qu'à leur tour. Ce fait indique les limites d'un système de sécurité intérieure purement privé : l'appareil répressif de l'État reste nécessaire pour minimiser les inégalités devant le crime en protégeant tout le monde, les pauvres comme les riches.

Cependant, il est tout simplement faux de dire que les bénéfices de la sécurité privếe sont réservés aux riches. La plupart des serrures sont à la portée de toutes les bourses. Récemment, on mettait sur le marché des alarmes portatives peu coûteuses. La baisse généralisée des prix dans l'électronique rend les dispositifs de sécurité de plus en plus abordables. Plus important encore, les pauvres profitent, indirectement mais très réellement, de services de sécurité chaque fois qu'ils fréquentent les supermarchés, les centres

3. Sur le déplacement et la diffusion des bénéfices de la prévention, voir Clarke et Mayhew, 1988 ; Gabor, 1990 ; Clarke et Weiseburd, 1994.

4. Selon Christie (1993: 108), la police privée qui protège les riches réduit chez ces derniers la motivation à payer pour une police publique dont les pauvres profiteraient : « $\mathrm{A}$ private police, caring for those able and willing to pay might reduce the interest among the upper classes in having a good public police... and thus leave the other classes and the inner cities in a even worse situation ". L'hypothèse ne manque pas de vraisemblance. Elle suppose cependant que Christie reconnaisse qu'une bonne police publique est possible et qu'elle contribue, par son activité normale, à la sécuritê des classes inférieures, réduisant ainsi les inégalités entre les riches et les pauvres. 
commerciaux, les transports en commun et les hôpitaux. Dans tous ces lieux, ils jouissent d'une protection et d'une tranquillité que l'on doit en partie aux intervenants en sécurité. Dans les usines et dans toutes sortes d'entreprises, les travailleurs, y compris les plus modestes, sont aussi protégés grâce à des mesures mises en place par le secteur privé. Bref, s'il est vrai que la sécurité privée est plus accessible aux riches qu'aux pauvres, ces derniers en profitent aussi à titre d'acheteurs, de clients, de travailleurs et de visiteurs dans les lieux semi-publics.

\section{Des gardes au-dessus de tout soupçon ?}

Le motif d'inquiétude le mieux fondé a trait à la qualité du personnel de base. Les gardes de sécurité sont-ils suffisamment compétents et intègres ? Les clients des agences de sécurité à contrat profitent de bas tarifs, mais ils n'en ont que pour leur argent. Les dirigeants des agences ne peuvent pas se permettre d'être regardants à l'embauche s'ils n'offrent pas des salaires alléchants. Qui plus est, les horaires de travail des gardes sont souvent impossibles et les tâches fastidieuses. Tout cela engendre un fort roulement du personnel et des difficultés de recrutement. Les raisons de s'inquiéter sont fondées. Le gardien qui a accès à du matériel confidentiel ou à des objets précieux est en position pour commettre des vols ou abuser de la confiance placée en lui. En l'absence d'une sélection rigoureuse du personnel, on court le risque d'embaucher des individus d'une moralité douteuse ou même des délinquants qui se feront engager précisément pour s'infiltrer là où ils peuvent commettre des délits (South, 1988). La célèbre question que posaient les Romains reste donc d'actualité : qui gardera nos gardiens?

\section{L'AVENIR : DEUX TENDANCES}

Quoi qu'il en soit, le stéréotype de la sécurité privée incarné par le gardien ignare et mal payé est de plus en plus dépassé. En effet, deux évolutions récentes contribuent à modifier la physionomie de la sécurité privée : l'intégration croissante des technologies et le développement d'une expertise de plus en plus fine.

\section{L'intégration des technologies}

Les années 1990 sont marquées par un recul des effectifs des gardes de sécurité compensé par une utilisation croissante des technologies et, surtout, par leur intégration. Cette tendance a été soulignée par Brodeur (1995). Aux États-Unis et en Grande-Bretagne, le nombre de gardes diminue, cependant qu'augmentent les ventes d'équipements les plus divers (pour le Québec, voir Degailler dans le présent numéro). 
Le dynamisme de la technologie de sécurité éclate dans trois secteurs : 1) les systèmes d'alarmes et de détection (détecteurs à infrarouge, à microondes, à ultrasons, détecteurs de métaux, détecteurs électromagnétiques, étiquettes électroniques, détecteurs de drogues, centrales d'alarmes);2) les systèmes de télésurveillance (TVCF, caméras de surveillance, vidéosurveillance, caméras cachées, centrales de surveillance) ; 3) les systèmes de contrôles d'accès (identification par cartes magnétiques, cartes à puces, cartes de proximité, centrales de contrôles d'accès).

L'évolution la plus remarquable est l'intégration de plus en plus poussée d'équipements variés grâce aux technologies de la communication, de l'électronique et de l'informatique. Une centrale unique de télésurveillance, d'alarme et de contrôle d'accès peut recevoir des signaux de plusieurs sources différentes : détecteurs, caméras, accès contrôlés par cartes à puces, téléphone, gardes... Des micro-ordinateurs équipés de logiciels spécialisés aident alors le responsable de la sécurité à traiter l'information très rapidement et à obtenir la réponse appropriée. Qui plus est, les événements et les interventions étant enregistrés, ils peuvent être stockés sur support informatique et classés pour fins d'analyses périodiques destinées à découvrir les problèmes récurrents et à évaluer l'impact des solutions adoptées dans le passé.

Cependant, les limites de la technologie et ses inconvénients sont indiscutables, comme en témoigne la plaie des fausses alarmes : non seulement elles dérangent, mais elles émoussent la vigilance en criant au loup électroniquement (Felson, 1997). Comme n'importe quel outil, les équipements de sécurité ne valent que s'ils sont utilisés correctement. Les systèmes de télésurveillance ne sont utiles que combinés à l'intelligence et à l'intervention humaine. S'ils sont installés sans étude préalable des besoins et du site, si les gardiens que l'on place devant les moniteurs ne sont ni attentifs ni perspicaces et si les détections ne sont pas suivies d'interventions, ces systèmes se révéleront des investissements aussi inutiles que coûteux, comme on l'a constaté dans le métro de Paris (Boullier, 1995) et de Montréal (Grandmaison et Tremblay, 1994). Par contre, ce type de matériel est performant quand il est adéquat et judicieusement installé, quand les surveillants des moniteurs sont perspicaces et quand l'intervention suit.

\section{Une expertise fondée sur l'intelligence des problèmes}

Les limites de la technologie ont conduit les meilleurs consuitants en sécurité à miser plutôt sur une analyse fine des problèmes et sur une alliance avec les demandeurs de sécurité (y compris le personnel travaillant sur le site qu'il s'agit de protéger). C'est ainsi que, dans les hôpitaux de Paris, la sécurité est gérée avec d'excellents résultats en misant moins sur la technologie que sur l'intelligence des problèmes par des chefs de sécurité compétents (voir Le Doussal, 1991, 1995a et b ; Le Doussal, et Laures-Colonna, 1992). 
Les hôpitaux de Paris qui relèvent de l'Assistance publique forment un vaste réseau : 51 hôpitaux, 30000 lits, 200000 admissions par an. Ils sont ouverts au public. Réagissant à la persistance du sentiment d'insécurité dans leurs établissements, les directeurs de ces hôpitaux décident à la fin des années 1980 de procéder à des audits de sécurité pour y voir plus clair avant d'agir. Terminés en 1990, ces audits concluent que la nature et l'ampleur de l'insécurité réelle sont très mal connues : les incidents et les actes de malveillance ne sont pas enregistrés systématiquement ; ceux qui le sont font l'objet d'un traitement au cas par cas. La malveillance n'est donc ni mesurée, ni décrite, ni analysée. Sa spécificité est ignorée. On l'assimile à d'autres risques : accidents, incendies ...

Les premiers dénombrements rigoureux apprennent aux auditeurs que les crimes violents commis dans les hôpitaux sont rares ( $5 \%$ de l'ensemble des actes de malveillance relevés). En revanche, les vols paraissent fréquents ( $85 \%$ des actes de malveillance) et représentent le vecteur principal de l'insécurité. Les auditeurs constatent que de nombreux vols d'occasion sont commis dans les chambres, donnant aux patients et au personnel le sentiment que d'inquiétants rôdeurs errent en toute liberté dans l'hôpital. Un examen attentif des incidents révèle que des voleurs récidivistes vont d'un hôpital à l'autre, sous des déguisements divers et avec des prétextes variés, pour commettre des vols en grand nombre. Un vol sur deux est le fait de ces rats d'hôpitaux. Ils dérobent des effets personnels et des porte-monnaie dans les chambres de malades, s'introduisent dans les vestiaires, dans les locaux de soins et dans les véhicules (Le Doussal et Laures-Colonna, 1992 ; Le Doussal, $1995 \mathrm{a}$ et b).

Il est alors décidé que la lutte contre le vol deviendra la priorité de la sécurité antimalveillance. Il s'agit d'en réduire le nombre de façon « efficace, durable, peu coûteuse et sans gêner le fonctionnement de l'hôpital, tout en lui conservant sa vocation de lieu ouvert et de lieu de confiance " (Le Doussal, 1995b : 596). La recherche de solutions est guidée par un souci de spécificité : la lutte contre la malveillance pose des problèmes distincts de la sécurité technique ou de la prévention des incendies. Il ne s'agit pas de prévenir des accidents mais de lutter contre une activité intentionnelle qui évolue, s'adapte et déjoue les protections mises en place pour la contrer. On en déduit la nécessité de faire de la sécurité anti-malveillance un métier distinct de la sécurité-incendie. À cette fin, il est décidé de créer un poste de chef de la sécurité anti-malveillance au sein de l'hôpital. Pour le combler, l'on recrute d'anciens policiers ou gendarmes triés sur le volet. Un chef de la sécurité est attaché à un hôpital et il reçoit la mission de travailler en étroite collaboration avec le directeur général et le personnel. On lui demande d'abord de connaître à fond le site et les actes de malveillance qui s'y 
produisent. Il aura pour tâche d'élaborer une politique de prévention, de mener des investigations sur les circonstances de tous les vols, d'aider les victimes et d'acheminer les plaintes à la police. Il est aussi appelé à conseiller le directeur hospitalier, notamment sur la dimension sécuritaire des projets de construction et de rénovation, et à soutenir le personnel hospitalier dans ses efforts de prévention de la malveillance. Enfin, il lui revient d'évaluer l'efficacité des technologies de sécurité sur le site, des prestations de gardiennage et de ses propres actions (Le Doussal, 1995b).

La reconnaissance de la contingence des problèmes est au cceur du dispositif : « la prévention des vols n'est pas une juxtaposition de recettes qu'on pourrait systématiquement appliquer à des situations prédéfinies. Elle nécessite au contraire, dans le cadre d'une stratégie globale, une réflexion adaptée à chaque situation » (Le Doussal, 1995 : 601). C'est la raison pour laquelle un nouveau chef de la sécurité a trois mois pour se familiariser avec le site qu'il est appelé à défendre et pour connaître les problèmes qui s'y posent. Il doit en tirer un rapport préliminaire dans lequel il fera un état de la situation et ébauchera un plan d'action.

Le chef de la sécurité recense l'information sur tous les actes de malveillance commis dans l'hôpital. Il mène une enquête sur chacun d'eux. Ce travail d'enquête ne lui sert pas seulement à identifier des coupables, il lui permet aussi, à la longue, d'accumuler les informations dont il a besoin pour se faire une idée d'ensemble des menaces qui pèsent sur le site. Il pourra ainsi élaborer une politique de prévention fondée sur une connaissance précise du problème à résoudre.

Finalement, le chef de la sécurité a pour mandat de travailler en étroite collaboration avec le personnel hospitalier. Il gagne sa confiance par sa compétence spécifique, par son respect de la culture hospitalière et par son souci des droits individuels. Cela le conduit à mettre l'accent sur la prévention situationnelle, la répression étant jugée menaçante par le personnel hospitalier.

Cette politique produit des résultats remarquables. À la fin de la première année de présence d'un chef de sécurité dans un hôpital, les vols y baissent de 30 à $60 \%$ du nombre enregistrés six mois auparavant. Durant les années suivantes, la baisse est environ de $10 \%$ par année. Entre 1990 et 1994, le total des vols commis dans 50 hôpitaux de Paris passe de 12000 à 6000 (Le Doussal, 1995b : 596). Ce degré d'efficacité est d'abord l'effet d'un système d'action stratégique fondé sur la connaissance des problèmes. Y arriver suppose : 1) des chefs de sécurité compétents ; 2) une mission précise reconnaissant la spécificité de la malveillance ; 3) la confiance et la collaboration de la direction et du personnel ; 4) la connaissance du site et de ses menaces ; 5) une attitude proactive vis-à-vis des voleurs. 
Ce succès montre qu'il n'est pas vain d'espérer obtenir à un prix raisonnable une sécurité efficace sans pour autant interférer dans la vocation de l'établissement qu'il s'agit de protéger. Tout l'art est de créer une synergie entre les quatre ressources pouvant être mises au service de la sécurité : 1) la technologie ; 2) les gardes de sécurité ; 3) le personnel œuvrant sur le site ; 4) les lieux physiques dont l'aménagement permet de faciliter la surveillance et les contrôles. Cet art présuppose cependant une connaissance raisonnée des problèmes, du site et de l'adversaire. Il ne s'agit pas de déployer à l'aveuglette le matériel et les hommes mais de miser sur l'intelligence - le mot étant compris autant dans son sens anglais de renseignement que français de maîtrise intellectuelle des situations. L'adaptation symbiotique des mesures de sécurité à un site et aux besoins des gens qui s'y trouvent représente un défi pour l'esprit. Il s'agit d'appréhender la nature précise de la demande, de connaître les lieux, les personnes et les opérations pour ensuite imaginer une stratégie qui combine, dans un tout homogène, les personnes et l'équipement.

L'intelligence des situations s'impose aussi parce qu'un dispositif sécuritaire doit être accepté par les intéressés et être compatible avec le fonctionnement de l'organisation dans laquelle on l'installe. Il n'est pas évident de fournir au client une sécurité abordable avec un minimum de désagrément. Si un équipement $s$ 'avère être une nuisance, on le rendra vite inopérant - parfois même en le sabotant - puis il sera rejeté comme un corps étranger : les alarmes seront désarmées, les portes déverrouillées et les moniteurs vidéo laissés sans surveillance. Il faut de la finesse pour permettre la libre circulation des personnes tout en filtrant les intrus; pour respecter l'intimité des gens tout en ayant les malveillants à l'cil ; pour éviter les fausses alarmes tout en détectant les intrusions.

Pour connaître les données du problème de sécurité qui se pose sur un site, la méthodologie d'analyse développée en prévention du crime serait utilisée avec profit. Elle préconise de recueillir systématiquement des données : 1) sur les délits dont un site est le théâtre ;2) sur ses auteurs ;3) sur ses victimes ; 4) sur ses causes ; 5) sur les situations pre-criminelles ; 6) sur les dispositifs de sécurité et de contrôle déjà en place ; 7) sur les vulnérabilités des personnes et des objets. C'est en s'appuyant sur une analyse de ces renseignements qu'une stratégie de prévention taillée sur mesure peut être conçue, mise en œuvre et évaluée (Cusson, Tremblay, Biron, Ouimet et Grandmaison, 1994).

En la matière, la quantification est utile pour prendre la mesure exacte des problèmes et pour évaluer l'impact des solutions. Elle devient de plus en plus facile grâce aux ordinateurs : les incidents enregistrés par les gardes, les détecteurs et les caméras sont mis en mémoire pour être ensuite analysés à l'aide des logiciels idoines. Dans les commerces et autres entreprises vivant 
du profit, la quantification des pertes dues au vol, d'une part, et des gains dus à la prévention, d'autre part, servirait à persuader les commerçants qu'il est rentable d'investir dans la prévention. Actuellement, ils hésitent à y consacrer des ressources, ne connaissant pas l'ampleur des pertes que leur cause le vol. Or, il se développe une méthodologie pour estimer les coûts dus au vol dans les entreprises (Challinger, 1997 ; Hollinger, 1997). Jumelée aux méthodologies d'évaluation en prévention du crime, elle permettrait de chiffrer les économies qu'une entreprise réaliserait grâce à un programme de prévention.

Une telle démarche ne peut être menée à bien que par des spécialistes formés et exercés à traiter une information pas toujours transparente, et capables de concevoir un train intégré de mesures. En définitive, on ne voit pas comment le marché de la sécurité pourra faire l'économie d'experts qui sauront penser la sécurité.

\section{RÉFÉRENCES}

BELLOT, C. et COUSINEAU, M.-M. (1996), «Le métro : espace de vie, espace de contrôle », Déviance et société, vol. 20, nº 4, p. 377-395.

BOULLIER, D. (1995), " La vidéosurveillance à la RATP : un maillon controversé de la chaîne de production de sécurité ", Les Cahiers de la sécurité intérieure, $\mathrm{n}^{\circ} 21$, p. 88 100.

BRODEUR, J.-P. (1995), « Le contrôle social : privatisation et technocratie », Déviance et société, vol. $19, \mathrm{n}^{\circ} 2$, p. 127-147.

CHALLINGER, D. (1997), "Will crime prevention ever be a business priority? » in FELSON, M. et CLARKE, R.V. (dir.), Business and Crime Prevention, (p. 35-56), MONSEY, N.Y., Criminal Justice Press.

CHRISTIE, N. (1993), Crime Control as Industry, London, Routledge.

CLARKE, R. V. et MAYHEW, P. (1988), "The british gas suicide story and its criminological implication $\gg$ in TONRY, M. et MORRIS, N. (dir.), Crime and Justice, vol. 10, (p. 79-116), University of Chicago Press.

CLARKE, R. V. et WEISEBURD, D.L. (1994), « Diffusion of crime control benefits : observations on the reverse of displacement » in CLARKE, R.V. (dir.), Crime Prevention Studies, vol. 2, (p. 165-184), Monsey, N.Y., Criminal Justice Press.

CUSSON, M., TREMBLAY, P., BIRON, L., OUIMET, M. et GRANDMAISON, R. (1994), La planification et l'évaluation de projets en prévention du crime, Québec, Ministère de la Sécurité publique.

FELSON, M. (1997), « Technology, business and crime " in FELSON, M. et CLARKE, R.V. (dir.), Business and Crime Prevention, (p. 81-96), Monsey, N.Y., Criminal Justice Press.

FOURCAUDOT, M. (1988), Étude descriptive sur les agences de sécurité privée au Québec, Montréal, École de criminologie, Université de Montréal, mémoire de maitrise inédit.

GABOR, T. (1990), "Crime displacement and situational prevention : toward the development of some principles ", Canadian Joumal of Criminology, vol. 32, p. 41-74. 
GAGNON, I. (1995), Politiques et pratiques de renvoi au pénal : le cas d'une agence de sécurité privée à contrat, Montréal, École de criminologie, Université de Montréal, mémoire de maîtrise inédit.

GRANDMAISON, R. et TREMBLAY, P. (1994), Évaluation des effets de la télésurveillance sur la criminalité commise dans 13 stations de métro de Montréal, Montréal, Université de Montréal, Les Cahiers de l'École de criminologie.

HOLLINGER, R. C, (1997), "Measuring crime and its impact in the business environment » in FELSON, M. et CLARKE, R.V. (dir.), Business and Crime Prevention, (p. 5780), Monsey, N.Y., Criminal Justice Press.

LE DOUSSAL, R. (1991), «La sécurité privée dans un service public : un an d'expérience à l'Assistance publique », Les Cahiers de la sécurité intérieure, $\mathrm{n}^{\circ}$ 3, p. 113-130.

LE DOUSSAL, R. (1995a), «À l'hôpital : anti-malveillance et technologie », Les Cahiers de la sécurité intérieure, $\mathrm{n}^{\circ} 21$, p. 75-87.

LE DOUSSAL, R. (1995b), "La lutte contre les vols », Gestions Hospitalières, oct. 1995, p. 595-60t.

LE DOUSSAL, R. et LAURES-COLONNA, P. (1992), La sécurité à l'hôpital, Paris, Éditions ESF.

LOUBET DEL BAYLE, J.-L. (1992), La police : approche socio-politique, Paris, Montchrestien (coll. Clefs).

OCQUETEAU, F. et POTTIER, M. L. (1995a), Vigilance et sécurité dans les grandes surfaces, Paris, L'Harmattan-IHESI.

OCQUETEAU, F. et POTTIER, M. L. (1995b), «Vidéosurveillance et gestion de l'insécurité dans un centre commercial : les leçons de l'observation ", Les cahiers de la sécurité intérieure, $\mathrm{n}^{\mathrm{b}} 2 \mathrm{~L}$, p. 60-74.

PHILIPS, S. et COCHRANE, R. (1988), Crime and nuisance in the shopping center, London, Home Office, Crime Prevention Unit, paper 16.

POYNER, B. et WEBB, B. (1992), "Reducing theft from shopping bags in city " in CLARKE, R.V. (dir.), Situational Crime Prevention. Successful Cases Studies, (p. 99107). New York, Harrow and Heston.

REISS, A. (1987), « The legitimacy of intrusions into private space » in SHEARING, C. D et STENNING, P. C. (dir.), Private Policing, (p. 19-44), Newbury Park, California, Sage.

SHEARING, C.D. et STENNING, P. C. (1981), « Modern private security : its growth and implications » in TONRY, M. et MORRIS, N. (dir.), Crime and Justice, vol. 3. (p. 193246), Chicago, University of Chicago Press.

SOUTH, N. (1988), Policing for Profit, London, Sage.

TREMBLAY, S. et COUSINEAU, M.-M. (1996), « Why companies may not prosecute following security investigation », Canadian Security, vol. 18, n" 5, p. 22-23.

VAN DIJK, J. et TERLOUW, G. J. (1996), An internationat perspective of the business community as victims of fraud and crime, Pays-Bas, Ministère de la Justice. 